\title{
Higher Integrability for Very Weak Solutions of Inhomogeneous $A$-Harmonic Form Equations
}

\author{
Yuxia Tong, ${ }^{1,2}$ Shenzhou Zheng, ${ }^{1}$ and Jiantao $\mathbf{G u}^{2}$ \\ ${ }^{1}$ Department of Mathematics, Beijing Jiaotong University, Beijing 100044, China \\ ${ }^{2}$ College of Science, Hebei United University, Tangshan, Hebei 063009, China \\ Correspondence should be addressed to Yuxia Tong; tongyuxia@126.com
}

Received 5 February 2014; Revised 12 June 2014; Accepted 16 June 2014; Published 13 July 2014

Academic Editor: Hongya Gao

Copyright (c) 2014 Yuxia Tong et al. This is an open access article distributed under the Creative Commons Attribution License, which permits unrestricted use, distribution, and reproduction in any medium, provided the original work is properly cited.

The higher integrability for very weak solutions of $A$-harmonic form equations $d^{*} A(x, u, d u)=B(x, u, d u)$ has been proved.

\section{Introduction}

The aim of the present paper is to prove the higher integrability for very weak solutions of $A$-harmonic form equation

$$
d^{*} A(x, u, d u)=B(x, u, d u),
$$

with the more general growth conditions than (4); that is, we assume that $A: \Omega \times \bigwedge^{l-1}\left(\mathbf{R}^{n}\right) \times \Lambda^{l}\left(\mathbf{R}^{n}\right) \rightarrow \Lambda^{l}\left(\mathbf{R}^{n}\right)$, $B: \Omega \times \bigwedge^{l-1}\left(\mathbf{R}^{n}\right) \times \bigwedge^{l}\left(\mathbf{R}^{n}\right) \rightarrow \bigwedge^{l-1}\left(\mathbf{R}^{n}\right)$ satisfy the following conditions on a bounded convex domain $\Omega$ :

$$
\begin{gathered}
|A(x, u, \xi)| \leq \beta_{1}|\xi|^{p-1}+\gamma_{1}\left|u-u_{\Omega}\right|^{p-1}+f_{1}(x), \\
|B(x, u, \xi)| \leq \beta_{2}|\xi|^{s}+\gamma_{2}\left|u-u_{\Omega}\right|^{s}+f_{2}(x), \\
\langle A(x, u, \xi), \xi\rangle \geq \alpha|\xi|^{p} \\
A(x, u, \lambda \xi)=\lambda|\lambda|^{p-2} A(x, u, \xi),
\end{gathered}
$$

for almost every $x \in \Omega$, all $(l-1)$-differential forms $u$, and $l$-differential forms $\xi$. Here, $\alpha, \beta_{1}, \beta_{2}, \gamma_{1}, \gamma_{2}>0$ are positive constants, $0<s<p-1$, and $\max \{1, p-1\}<r<p<n$ is a fixed exponent associated with (1), the nonnegative functions $f_{1}, f_{2} \in L^{t /(p-1)}(\Omega)$ for $t>p$.

Definition 1. A differential form $u \in W_{\text {loc }}^{1, r}\left(\Omega, \bigwedge^{l-1}\right)$ with $\max \{1, p-1\}<r<p$ is called a very weak solution to (1) if $u$ satisfies

$$
\int_{\Omega}\langle A(x, u, d u), d \varphi\rangle \mathrm{d} x=\int_{\Omega} B(x, u, d u) \varphi \mathrm{d} x
$$

for all $\varphi \in W^{1, r /(r-p+1)}\left(\Omega, \bigwedge^{l-1}\right)$ with compact support.

The special type of (1) is

$$
d^{*} A(x, d u)=0,
$$

where $A: \Omega \times \bigwedge^{l}\left(\mathbf{R}^{n}\right) \rightarrow \bigwedge^{l}\left(\mathbf{R}^{n}\right)$ satisfies the conditions

$$
|A(x, \xi)| \leq \beta|\xi|^{p-1}, \quad\langle A(x, \xi), \xi\rangle \geq \alpha|\xi|^{p}
$$

for almost every $x \in \Omega$ and all $\xi \in \bigwedge^{l}\left(\mathbf{R}^{n}\right)$. Here, $\alpha, \beta>0$ are constants and $1<p<n$ is a fixed exponent associated with (4). $u \in W_{\text {loc }}^{1, p}\left(\Omega, \bigwedge^{l-1}\right)$ is an $A$-harmonic tensor in $\Omega$ if $u$ satisfies (4) in $\Omega$.

When $u$ is a 0 -form, that is, $u$ is a function, (1) is equivalent to

$$
\operatorname{div} A(x, u, \nabla u)=B(x, u, \nabla u) .
$$

Lots of results have been obtained in recent years about different versions of the $A$-harmonic equation; see [1-13].

In 1994, Iwaniec and Sbordone [3] first introduced weakly $A$-harmonic mapping. The word weak means that the integrable exponent $r$ of $u$ is smaller than the natural exponent p. In 1995, Stroffolini [14] gave the higher integrability result of weakly A-harmonic tensors. In 2010, Gao and Wang [15] 
gave an alternative proof of the higher integrability result of weakly $A$-harmonic tensors by introducing the definition of weak $W T_{2}$-class of differential forms.

In this paper, we continue to consider the higher integrability. To the generalized form of (1), under some general conditions (2) given above on the operator $A$, we obtain the higher integrability for very weak solutions to (1).

The following is our main results.

Theorem 2. Let $\Omega$ be a bounded convex domain of $\mathbf{R}^{n}$. There exist exponents $1<r_{1}=r_{1}\left(n, p, \beta_{1}, \beta_{2}\right)<p<r_{2}=$ $r_{2}\left(n, p, \beta_{1}, \beta_{2}\right)<\infty$ such that if $u \in W_{\text {loc }}^{1, r_{1}}\left(\Omega, \bigwedge^{l-1}\right)$ is a very weak solution of (1), then $u \in W_{\text {loc }}^{1, r_{2}}\left(\Omega, \bigwedge^{l-1}\right)$. In particular, $u \in W_{\text {loc }}^{1, p}\left(\Omega, \bigwedge^{l-1}\right)$ is a weak solution of $(1)$ in the usual sense.

Remark 3. To prove theorem, we have to estimate the integral of some power of $|u|$ and $|\varphi|$ by means of $|\nabla u|$ and $|\nabla \varphi|$, respectively. We deal with this difficulty by imbedding inequalities for differential forms. In addition, to reduce the integrable exponent of $d u$, we use Lemma 7.

\section{Notion and Lemmas}

We keep using the traditional notation.

Let $\Omega$ be a bounded convex domain of $\mathbf{R}^{n}$, let $e_{1}, e_{2}, \ldots, e_{n}$ be the standard unit basis of $\mathbf{R}^{n}$, and let $\bigwedge^{l}=\bigwedge^{l}\left(\mathbf{R}^{n}\right)$ be the linear space of $l$-covectors, spanned by the exterior products $e_{I}=e_{i_{1}} \wedge e_{i_{2}} \wedge \cdots \wedge e_{i_{l}}$, corresponding to all ordered $l$-tuples $I=\left(i_{1}, i_{2}, \ldots, i_{l}\right), 1 \leq i_{1}<i_{2}<\cdots<i_{l} \leq n, l=0,1, \ldots, n$. Let $\mathbf{R}=\mathbf{R}^{1}$. The Grassmann algebra $\Lambda=\oplus \bigwedge^{l}$ is a graded algebra with respect to the exterior products. For $\alpha=\sum \alpha^{I} e_{I} \in \bigwedge$ and $\beta=\sum \beta^{I} e_{I} \in \bigwedge$, the inner product in $\Lambda$ is given by $\langle\alpha, \beta\rangle=$ $\sum \alpha^{I} \beta^{I}$ with summation over all $l$-tuples $I=\left(i_{1}, i_{2}, \ldots, i_{l}\right)$ and all integers $l=0,1, \ldots, n$. The Hodge star operator $\star$ : $\bigwedge \rightarrow \bigwedge$ is denoted by rules $\star 1=e_{1} \wedge e_{2} \wedge \cdots \wedge e_{n}$ and $\alpha \bigwedge \star \beta=\beta \bigwedge \star \alpha=\langle\alpha, \beta\rangle(\star 1)$ for all $\alpha, \beta \in \bigwedge$. The norm of $\alpha \in \bigwedge$ is given by formula $|\alpha|^{2}=\langle\alpha, \alpha\rangle=\star(\alpha \bigwedge \star \alpha) \epsilon$ $\Lambda^{0}=\mathbf{R}$. The Hodge star is an isometric isomorphism on $\Lambda$ with $\star: \bigwedge^{l} \rightarrow \bigwedge^{n-l}$ and $\star \star(-1)^{l(n-l)}: \bigwedge^{l} \rightarrow \bigwedge^{l}$. Balls are denoted by $B$ and $\rho B$ is the ball with the same center as $B$ and with $\operatorname{diam}(\rho B)=\rho \operatorname{diam}(B)$. We do not distinguish balls from cubes throughout this paper. The $n$-dimensional Lebesgue measure of a measurable set $E \subseteq \mathbf{R}^{n}$ is denoted by $|E|$.

Differential forms are important generalizations of real functions and distributions; note that a 0 -form is the usual function in $\mathbf{R}^{n}$. A differential $l$-form $\omega$ on $\Omega$ is a Schwartz distribution on $\Omega$ with values in $\bigwedge^{l}\left(\mathbf{R}^{n}\right)$. We use $D^{\prime}\left(\Omega, \Lambda^{l}\right)$ to denote the space of all differential $l$-forms $\omega(x)=$ $\sum_{I} \omega_{I}(x) d x_{I}=\sum \omega_{i_{1} i_{2} \cdots i_{l}}(x) d x_{i_{1}} \wedge d x_{i_{2}} \wedge \cdots \wedge d x_{i_{l}}$. We write $L^{p}\left(\Omega, \bigwedge^{l}\right)$ for the $l$-forms with $\omega_{I} \in L^{p}(\Omega, \mathbf{R})$ for all ordered $l$-tuples $I$. Thus, $L^{p}\left(\Omega, \bigwedge^{l}\right)$ is a Banach space with norm

$$
\begin{aligned}
\|\omega\|_{p, \Omega} & =\left(\int_{\Omega}|\omega(x)|^{p} d x\right)^{1 / p} \\
& =\left(\int_{\Omega}\left(\sum\left|\omega_{I}(x)\right|^{2}\right)^{p / 2} d x\right)^{1 / p} .
\end{aligned}
$$

For $\omega \in D^{\prime}\left(\Omega, \Lambda^{l}\right)$, the vector-valued differential form $\nabla \omega=\left(\partial \omega / \partial x_{1}, \ldots, \partial \omega / \partial x_{n}\right)$ consists of differential forms $\partial \omega / \partial x_{i} \in D^{\prime}\left(\Omega, \Lambda^{l}\right)$ where the partial differentiations are applied to the coefficients of $\omega$. As usual, $W^{1, p}\left(\Omega, \bigwedge^{l}\right)$ is used to denote the Sobolev space of $l$-forms, which equals $L^{p}\left(\Omega, \bigwedge^{l}\right) \cap L_{1}^{p}\left(\Omega, \bigwedge^{l}\right)$ with norm

$$
\begin{aligned}
\|\omega\|_{W^{1, p}\left(\Omega, \wedge^{l}\right)} & =\|\omega\|_{W^{1, p}\left(\Omega, \Lambda^{l}\right)} \\
& =\operatorname{diam}(\Omega)^{-1}\|\omega\|_{p, \Omega}+\|\nabla \omega\|_{p, \Omega} .
\end{aligned}
$$

The notations $W_{\text {loc }}^{1, p}(\Omega, \mathbf{R})$ and $W_{\text {loc }}^{1, p}\left(\Omega, \bigwedge^{l}\right)$ are selfexplanatory. We denote the exterior derivative by $d: D^{\prime}\left(\Omega, \bigwedge^{l}\right) \rightarrow D^{\prime}\left(\Omega, \bigwedge^{l+1}\right)$ for $l=0,1, \ldots, n$. Its formal adjoint operator $d^{\star}: D^{\prime}\left(\Omega, \Lambda^{l+1}\right) \rightarrow D^{\prime}\left(\Omega, \Lambda^{l}\right)$ is given by $d^{\star}=(-1)^{n l+1} \star d \star$ on $D^{\prime}\left(\Omega, \bigwedge^{l+1}\right), l=0,1, \ldots, n$. A differential $l$-form $u \in D^{\prime}\left(\Omega, \bigwedge^{l}\right)$ is called a closed form if $d u=0$ in $\Omega$. It is called exact if there exists a differential form $\alpha \in D^{\prime}\left(\Omega, \bigwedge^{l-1}\right)$ such that $u=d \alpha$. Poincaré lemma implies that exact forms are closed.

From $[1,16]$, if $D \subset \mathbf{R}^{n}$ is a bounded convex domain, to each $y \in D$, there corresponds a linear operator $K_{y}$ : $C^{\infty}\left(D, \bigwedge^{l}\right) \rightarrow C^{\infty}\left(D, \bigwedge^{l-1}\right)$ defined by

$$
\begin{aligned}
& \left(K_{y} \omega\right)\left(x ; \xi_{1}, \ldots, \xi_{l-1}\right) \\
& \quad=\int_{0}^{1} t^{l-1} \omega\left(t x+y-t y ; x-y, \xi_{1}, \ldots, \xi_{l-1}\right) d t
\end{aligned}
$$

and a decomposition $\omega=d\left(K_{y} \omega\right)+K_{y}(d \omega)$. A homotopy operator $T: C^{\infty}\left(D, \bigwedge^{l}\right) \rightarrow C^{\infty}\left(D, \bigwedge^{l-1}\right)$ is defined by averaging $K_{y}$ over all points $y$ in $D$; that is,

$$
T \omega=\int_{D} \varphi(y) K_{y} \omega d y
$$

where $\varphi \in C_{0}^{\infty}(D)$ is normalized by $\int_{D} \varphi(y) d y=1$. Then, there is also a decomposition

$$
\omega=d(T \omega)+T(d \omega) .
$$

The $l$-form $\omega_{D} \in D^{\prime}\left(D, \bigwedge^{l}\right)$ is defined by

$$
\omega_{D}= \begin{cases}|D|^{-1} \int_{D} \omega(y) d y & \text { if } l=0 \\ d(T \omega) & \text { if } l=1,2, \ldots, n\end{cases}
$$

for all $\omega \in L^{p}\left(D, \bigwedge^{l}\right)$. Clearly, $\omega_{D}$ is a closed form and, for $l>0, \omega_{D}$ is an exact form.

We need the following lemmas.

Lemma 4 (see $[16,17])$. Let $\omega \in D^{\prime}\left(D, \bigwedge^{l}\right)$ be such that $d \omega \in$ $L^{p}\left(D, \bigwedge^{l+1}\right)$; then, $\omega-\omega_{D}$ is in $W^{1, p}\left(D, \bigwedge^{l}\right)$, and

$$
\left\|\omega-\omega_{D}\right\|_{p, D} \leq C(n, p) \operatorname{diam}(D)\|d \omega\|_{p, D}
$$

holds for a cube or a ball $D$ in $\mathbf{R}^{n}, l=0,1, \ldots, n$, and $1<p<$ $\infty$. 
Lemma 5 (see [18]). Suppose $X$ and $Y$ are vectors of an inner product space. Then,

$$
\left.|| X\right|^{\varepsilon} X-|Y|^{\varepsilon} Y\left|\leq \frac{1-\varepsilon}{1+\varepsilon} 2^{-\varepsilon}\right| X-\left.Y\right|^{1+\varepsilon},
$$

for $-1<\varepsilon \leq 0$, and

$$
\left.|| X\right|^{\varepsilon} X-|Y|^{\varepsilon} Y\left|\leq(1+\varepsilon)(|Y|+|X-Y|)^{\varepsilon}\right| X-Y \mid,
$$

for $\varepsilon \geq 0$.

Lemma 6 (see [14]). Let $D$ be a cube or a ball, and $\omega \epsilon$ $L^{s}\left(D, \bigwedge^{l}\right)$ with $d \omega \in L^{s}\left(D, \bigwedge^{l+1}\right)$. Then,

$$
\begin{aligned}
& \frac{1}{\operatorname{diam} D}\left(f_{D}\left|\omega-\omega_{D}\right|^{s}\right)^{1 / s} \\
& \quad \leq C(n, s)\left(f_{D}|d \omega|^{n s /(n+s-1)}\right)^{(n+s-1) / n s} .
\end{aligned}
$$

Here, we denote by $f_{D}$ the integral mean over $D$.

Lemma 7 (see [19], page 122, and Proposition 1.1). Let $Q$ be an n-cube. Suppose

$$
\begin{aligned}
& f_{\mathrm{Q}_{R}\left(x_{0}\right)}|g|^{q} d x \leq b\left(f_{\mathrm{Q}_{2 R}\left(x_{0}\right)}|g| d x\right)^{q} \\
& +f_{\mathrm{Q}_{2 R}\left(x_{0}\right)}|f|^{q} d x+\theta \int_{\mathrm{Q}_{2 R}\left(x_{0}\right)}|g|^{q} d x
\end{aligned}
$$

for each $x_{0} \in Q$ and each $R<(1 / 2) \operatorname{dist}\left(x_{0}, \partial Q\right)=R_{0}$, where $R_{0}, b, \theta$ are constants with $b>1, R_{0}>0,0 \leq \theta<1$. Then, $g(x) \in L_{\mathrm{loc}}^{p}(Q)$ for $p \in[q, q+\varepsilon)$ and

$$
\begin{aligned}
& \left(f_{Q_{R}}|g|^{p} d x\right)^{1 / p} \\
& \quad \leq C\left\{\left(f_{Q_{2 R}}|g|^{q} d x\right)^{1 / q}+\left(f_{Q_{2 R}}|f|^{p} d x\right)^{1 / p}\right\}
\end{aligned}
$$

for $Q_{2 R} \subset Q, R<R_{0}$, where $C$ and $\varepsilon$ are positive constants depending only on $b, \theta, q, n$.

\section{Proof of Theorem 2}

Let $u \in W_{\text {loc }}^{1, r}\left(\Omega, \bigwedge^{l-1}\right)$ be a very weak solution of (1) and let $Q(2 R) \subset \Omega$ be a cube. Fix a cutoff function $\eta(x) \in C_{0}^{\infty}(Q(2 R))$ such that $0 \leq \eta \leq 1,|\nabla \eta| \leq C(n) / R$, and $\eta \equiv 1$ on $Q(R)$. Adopting a usual convention, $C$ will denote a constant whose value may change in any two occurrences, and only the relevant dependences will be specified, as, for example, in $C(n)$.

Step 1. In order to take a suitable test form in the weak solutions of (1), we do a Hodge decomposition [16, 17] to distribution tensors fields $\left|d\left(\eta\left(u-u_{\mathrm{Q}(2 R)}\right)\right)\right|^{r-p} d(\eta(u-$ $\left.\left.u_{\mathrm{Q}(2 R)}\right)\right) \in L^{r /(r-p+1)}\left(Q(2 R), \Lambda^{l}\right)$. With the aid of Hodge decomposition,

$$
\left|d\left(\eta\left(u-u_{\mathrm{Q}(2 R)}\right)\right)\right|^{r-p} d\left(\eta\left(u-u_{\mathrm{Q}(2 R)}\right)\right)=d \varphi+h,
$$

where $d \varphi, h \in L^{r /(r-p+1)}\left(Q(2 R), \bigwedge^{l}\right)$, and

$$
\|h\|_{r /(r-p+1)} \leq C(n)(p-r)\left\|d\left(\eta\left(u-u_{\mathrm{Q}(2 R)}\right)\right)\right\|_{r}^{r-p+1} ;
$$

then, we have

$$
\begin{aligned}
\|d \varphi\|_{r /(r-p+1)} \\
\leq\left\|\left.d\left(\eta\left(u-u_{\mathrm{Q}(2 R)}\right)\right)\right|^{r-p} d\left(\eta\left(u-u_{\mathrm{Q}(2 R)}\right)\right)\right\|_{r /(r-p+1)} \\
+\|h\|_{r /(r-p+1)} \leq\left\|d\left(\eta\left(u-u_{\mathrm{Q}(2 R)}\right)\right)\right\|_{r}^{r-p+1} \\
\quad+C(n)(p-r)\left\|d\left(\eta\left(u-u_{\mathrm{Q}(2 R)}\right)\right)\right\|_{r}^{r-p+1} \\
\leq C(n)\left\|d\left(\eta\left(u-u_{\mathrm{Q}(2 R)}\right)\right)\right\|_{r}^{r-p+1} .
\end{aligned}
$$

For $d \varphi \in L^{r /(r-p+1)}\left(Q(2 R), \bigwedge^{l}\right)$, it is clear that $\varphi-\varphi_{\mathrm{Q}(2 R)} \in$ $W^{1, r /(r-p+1)}\left(Q(2 R), \bigwedge^{l-1}\right)$ by Lemma 4 . We can use $\varphi-$ $\varphi_{\mathrm{Q}(2 R)} \in W^{1, r /(r-p+1)}\left(Q(2 R), \bigwedge^{l-1}\right)$ as a test form for (1). Let

$$
\begin{aligned}
E= & \left|d\left(\eta\left(u-u_{\mathrm{Q}(2 R)}\right)\right)\right|^{r-p} d\left(\eta\left(u-u_{\mathrm{Q}(2 R)}\right)\right) \\
& -\left|\eta d\left(u-u_{\mathrm{Q}(2 R)}\right)\right|^{r-p} \eta d\left(u-u_{\mathrm{Q}(2 R)}\right) .
\end{aligned}
$$

Combining the above formula with (19), we get

$$
\begin{aligned}
d & \left(\varphi-\varphi_{\mathrm{Q}(2 R)}\right) \\
& =d \varphi \\
& =\left|d\left(\eta\left(u-u_{\mathrm{Q}(2 R)}\right)\right)\right|^{r-p} d\left(\eta\left(u-u_{\mathrm{Q}(2 R)}\right)\right)-h \\
& =E-h+\left|\eta d\left(u-u_{\mathrm{Q}(2 R)}\right)\right|^{r-p} \eta d\left(u-u_{\mathrm{Q}(2 R)}\right) .
\end{aligned}
$$

Then, by Definition 1 ,

$$
\begin{aligned}
& \int_{\mathrm{Q}(2 R)}\langle A(x, u, d u), \\
& \left.E-h+\left|\eta d\left(u-u_{\mathrm{Q}(2 R)}\right)\right|^{r-p} \eta d\left(u-u_{\mathrm{Q}(2 R)}\right)\right\rangle \mathrm{d} x \\
& =\int_{\mathrm{Q}(2 R)} B(x, u, d u)\left(\varphi-\varphi_{\mathrm{Q}(2 R)}\right) \mathrm{d} x .
\end{aligned}
$$

That is,

$$
\begin{aligned}
& \int_{\mathrm{Q}(2 R)}\left\langle A(x, u, d u),\left|\eta d\left(u-u_{\mathrm{Q}(2 R)}\right)\right|^{r-p}\right. \\
& \left.\times \eta d\left(u-u_{\mathrm{Q}(2 R)}\right)\right\rangle d x \\
& =\int_{\mathrm{Q}(2 R)}\langle A(x, u, d u), h\rangle d x \\
& \quad-\int_{\mathrm{Q}(2 R)}\langle A(x, u, d u), E\rangle d x \\
& \quad+\int_{\mathrm{Q}(2 R)} B(x, u, d u)\left(\varphi-\varphi_{\mathrm{Q}(2 R)}\right) d x \\
& =I_{1}+I_{2}+I_{3} .
\end{aligned}
$$


Step 2. In this part, we are devoted to estimate every integration in (25), respectively. In the following, we will especially be concerned about the coefficient of $\int_{Q(2 R)}|d u|^{r} d x$. In our case, $r$ is sufficiently close to $p$. We can estimate $C(n, p, r,|\Omega|)$ independently of $r$; then, we will write constants $C(n, p, r, s,|\Omega|)=C(n, p, s,|\Omega|)=C$.

Noticing that $u_{\mathrm{Q}(2 R)}$ satisfies $d u_{\mathrm{Q}(2 R)}=0$, then by condition (2), the left integration in (25) becomes

$$
\begin{aligned}
& \int_{Q(2 R)}\langle A(x, u, d u), \\
& \left.\quad\left|\eta d\left(u-u_{Q(2 R)}\right)\right|^{r-p} \eta d\left(u-u_{Q(2 R)}\right)\right\rangle d x \\
& =\int_{Q(2 R)}\left\langle A(x, u, d u),|\eta d u|^{r-p} \eta d u\right\rangle d x \\
& =\int_{Q(2 R)}|\eta d u|^{r-p} \eta\langle A(x, u, d u), d u\rangle d x \\
& \geq \int_{Q(R)}|d u|^{r-p}\langle A(x, u, d u), d u\rangle d x \\
& \geq \alpha \int_{Q(R)}|d u|^{r} d x .
\end{aligned}
$$

In the following, we will estimate $I_{1}, I_{2}$, and $I_{3}$, respectively.

Estimate of $I_{1}$. By (2), Hölder's inequality, and (20),

$$
\begin{aligned}
& \left|I_{1}\right| \leq \int_{Q(2 R)}|A(x, u, d u)||h| d x \\
& \leq \int_{Q(2 R)}\left(\beta_{1}|d u|^{p-1}+\gamma_{1}\left|u-u_{Q(2 R)}\right|^{p-1}+f_{1}(x)\right)|h| d x \\
& \leq \beta_{1}\left(\int_{\mathrm{Q}(2 R)}|d u|^{r}\right)^{(p-1) / r} \\
& \times\left(\int_{Q(2 R)}|h|^{r /(r-p+1)} d x\right)^{(r-p+1) / r} \\
& +\gamma_{1}\left(\int_{Q(2 R)}\left|u-u_{Q(2 R)}\right|^{r}\right)^{(p-1) / r} \\
& \times\left(\int_{Q(2 R)}|h|^{r /(r-p+1)} d x\right)^{(r-p+1) / r} \\
& +\left(\int_{Q(2 R)}\left|f_{1}\right|^{r /(p-1)}\right)^{(p-1) / r} \\
& \times\left(\int_{Q(2 R)}|h|^{r /(r-p+1)} d x\right)^{(r-p+1) / r} \\
& \leq \beta_{1} C(n)(p-r)\left(\int_{Q(2 R)}|d u|^{r}\right)^{(p-1) / r} \\
& \times\left(\int_{\mathrm{Q}(2 R)}\left|d\left(\eta\left(u-u_{\mathrm{Q}(2 R)}\right)\right)\right|^{r} d x\right)^{(r-p+1) / r} \\
& +\gamma_{1} C(n)(p-r)\left(\int_{Q(2 R)}\left|u-u_{Q(2 R)}\right|^{r}\right)^{(p-1) / r}
\end{aligned}
$$

$$
\begin{aligned}
& \times\left(\int_{Q(2 R)}\left|d\left(\eta\left(u-u_{\mathrm{Q}(2 R)}\right)\right)\right|^{r} d x\right)^{(r-p+1) / r} \\
& +C(n)(p-r)\left(\int_{\mathrm{Q}(2 R)}\left|f_{1}\right|^{r /(p-1)}\right)^{(p-1) / r} \\
& \times\left(\int_{\mathrm{Q}(2 R)}\left|d\left(\eta\left(u-u_{\mathrm{Q}(2 R)}\right)\right)\right|^{r} d x\right)^{(r-p+1) / r} \\
& =I_{11}+I_{12}+I_{13} .
\end{aligned}
$$

Estimate of $I_{11}$. By Lemma 6 and by noticing that $\Omega$ is a bounded convex domain, we have

$$
\begin{aligned}
& \left(\int_{Q(2 R)}\left|u-u_{Q(2 R)}\right|^{r} d x\right)^{1 / r} \\
& \quad \leq C(n, r) R^{1 / r}\left(\int_{Q(2 R)}|d u|^{n r /(n+r-1)} d x\right)^{(n+r-1) / n r} \\
& \quad \leq C(n, r,|\Omega|)\left(\int_{Q(2 R)}|d u|^{n r /(n+r-1)} d x\right)^{(n+r-1) / n r}
\end{aligned}
$$

then, by

$$
\begin{aligned}
& \left(\int_{\mathrm{Q}(2 R)}\left|d\left(\eta\left(u-u_{\mathrm{Q}(2 R)}\right)\right)\right|^{r} d x\right)^{(r-p+1) / r} \\
& =\left(\int_{\mathrm{Q}(2 R)}\left|\eta d u+\left(u-u_{\mathrm{Q}(2 R)}\right) d \eta\right|^{r} d x\right)^{(r-p+1) / r} \\
& \leq C(p, r)\left(\int_{\mathrm{Q}(2 R)}|\eta d u|^{r} d x\right)^{(r-p+1) / r} \\
& \quad+C(p, r)\left(\int_{Q(2 R)}\left|\left(u-u_{\mathrm{Q}(2 R)}\right) d \eta\right|^{r} d x\right)^{(r-p+1) / r} \\
& \leq C(p, r)\left(\int_{Q(2 R)}|d u|^{r} d x\right)^{(r-p+1) / r} \\
& \quad+\frac{C(n, p, r,|\Omega|)}{R^{r-p+1}} \\
& \quad \times\left(\int_{Q(2 R)}^{\left.|d u|^{n r /(n+r-1)} d x\right)^{(n+r-1)(r-p+1) / n r}}\right.
\end{aligned}
$$

with Young's inequality

$$
\begin{array}{r}
a b \leq \frac{\tau a^{p}}{p}+\frac{\tau^{-p^{\prime} / p} b^{p^{\prime}}}{p^{\prime}}, \quad \frac{1}{p}+\frac{1}{p^{\prime}}=1, \\
\forall a, b>0, \quad \tau>0, \quad p>1,
\end{array}
$$


we have

$$
\begin{aligned}
\left|I_{11}\right|= & \beta_{1} C(n)(p-r) \\
& \times\left(\int_{\mathrm{Q}(2 R)}|d u|^{r}\right)^{(p-1) / r} \\
& \times\left(\int_{\mathrm{Q}(2 R)}\left|d\left(\eta\left(u-u_{\mathrm{Q}(2 R)}\right)\right)\right|^{r} d x\right)^{(r-p+1) / r} \\
\leq & \beta_{1} C(n, p, r)(p-r) \int_{\mathrm{Q}(2 R)}|d u|^{r} d x \\
& +\beta_{1} C(n, p, r,|\Omega|)(p-r) \tau \int_{\mathrm{Q}(2 R)}|d u|^{r} d x \\
& +\frac{\beta_{1} C(n, p, r,|\Omega|)(p-r) \tau^{-(p-1) /(r-p+1)}}{R^{r}} \\
& \times\left(\int_{\mathrm{Q}(2 R)}|d u|^{n r /(n+r-1)} d x\right)^{(n+r-1) / n} .
\end{aligned}
$$

Noticing that $r$ is sufficiently close to $p$, there exists a constant $\varepsilon^{\prime}=1 / 2$ such that $0<p-r \leq \varepsilon^{\prime}<1$. Then, we have $p-1<$ $(p-1) /(r-p+1) \leq(p-1) /\left(1-\varepsilon^{\prime}\right)$, and $\tau^{-(p-1) /(r-p+1)} \leq C$.

(31) becomes

$$
\begin{aligned}
\left|I_{11}\right| \leq & \beta_{1} C(p-r)(1+\tau) \int_{\mathrm{Q}(2 R)}|d u|^{r} d x \\
& +\frac{\beta_{1} C(p-r)}{R^{r}}\left(\int_{\mathrm{Q}(2 R)}|d u|^{n r /(n+r-1)} d x\right)^{(n+r-1) / n} .
\end{aligned}
$$

Estimate of $I_{12}$. By Lemma 4 and noticing that $\Omega$ is a bounded convex domain, we have

$$
\begin{aligned}
& \left(\int_{\mathrm{Q}(2 R)}\left|u-u_{\mathrm{Q}(2 R)}\right|^{r} d x\right)^{1 / r} \\
& \quad \leq C(n, r) R\left(\int_{Q(2 R)}|d u|^{r} d x\right)^{1 / r} \\
& \quad \leq C(n, r,|\Omega|)\left(\int_{\mathrm{Q}(2 R)}|d u|^{r} d x\right)^{1 / r} ;
\end{aligned}
$$

then, by the above inequality, (29), and Young's inequality,

$$
\begin{aligned}
\left|I_{12}\right|= & \gamma_{1} C(n)(p-r) \\
& \times\left(\int_{\mathrm{Q}(2 R)}\left|u-u_{\mathrm{Q}(2 R)}\right|^{r}\right)^{(p-1) / r} \\
& \times\left(\int_{\mathrm{Q}(2 R)}\left|d\left(\eta\left(u-u_{\mathrm{Q}(2 R)}\right)\right)\right|^{r} d x\right)^{(r-p+1) / r} \\
\leq & \gamma_{1} C(n, p, r,|\Omega|)(p-r)\left(\int_{\mathrm{Q}(2 R)}|d u|^{r} d x\right)^{(p-1) / r} \\
& \times\left(\int_{\mathrm{Q}(2 R)}\left|d\left(\eta\left(u-u_{\mathrm{Q}(2 R)}\right)\right)\right|^{r} d x\right)^{(r-p+1) / r} \\
\leq & \gamma_{1} C(n, p, r,|\Omega|)(p-r) \int_{\mathrm{Q}(2 R)}|d u|^{r} d x \\
& +\gamma_{1} C(n, p, r,|\Omega|)(p-r) \tau \int_{\mathrm{Q}(2 R)}|d u|^{r} d x \\
& +\frac{\gamma_{1} C(n, p, r,|\Omega|)(p-r) \tau^{-(p-1) /(r-p+1)}}{R^{r}} \\
& \times\left(\int_{\mathrm{Q}(2 R)}|d u|^{n r /(n+r-1)} d x\right)^{(n+r-1) / n} \\
\leq & \gamma_{1} C(p-r)(1+\tau) \int_{\mathrm{Q}(2 R)}|d u|^{r} d x+\frac{\gamma_{1} C(p-r)}{R^{r}} \\
& \times\left(\int_{\mathrm{Q}(2 R)}|d u|^{n r /(n+r-1)} d x\right)^{(n+r-1) / n} \cdot \\
& (n)
\end{aligned}
$$

Estimate of $I_{13}$. By (31) and Young's inequality, we have

$$
\begin{aligned}
\left|I_{13}\right|= & C(n)(p-r) \\
& \times\left(\int_{\mathrm{Q}(2 R)}\left|f_{1}\right|^{r /(p-1)} d x\right)^{(p-1) / r} \\
& \times\left(\int_{\mathrm{Q}(2 R)}\left|d\left(\eta\left(u-u_{\mathrm{Q}(2 R)}\right)\right)\right|^{r} d x\right)^{(r-p+1) / r} \\
\leq & 2 C(n, p, r,|\Omega|)(p-r) \tau \int_{\mathrm{Q}(2 R)}\left|f_{1}\right|^{r /(p-1)} d x \\
& +C(n, p, r,|\Omega|)(p-r) \tau^{-(p-1) /(r-p+1)} \int_{\mathrm{Q}(2 R)}|d u|^{r} d x \\
& +\frac{C(n, p, r,|\Omega|)(p-r) \tau^{-(p-1) /(r-p+1)}}{R^{r}} \\
& \times\left(\int_{\mathrm{Q}(2 R)}|d u|^{n r /(n+r-1)} d x\right)^{(n+r-1) / n}
\end{aligned}
$$




$$
\begin{aligned}
\leq & 2 C(p-r) \tau \int_{Q(2 R)}\left|f_{1}\right|^{r /(p-1)} d x+C(p-r) \\
& \times \int_{Q(2 R)}|d u|^{r} d x \\
& +\frac{C(p-r)}{R^{r}}\left(\int_{Q(2 R)}|d u|^{n r /(n+r-1)} d x\right)^{(n+r-1) / n} .
\end{aligned}
$$

Combining (27), (31), (34), and (35) yields

$$
\begin{aligned}
\left|I_{1}\right| \leq & C(p-r)\left(\beta_{1}+\beta_{1} \tau+\gamma_{1}+\gamma_{1} \tau+1\right) \\
& \times \int_{Q(2 R)}|d u|^{r} d x \\
& +\frac{C(p-r)}{R^{r}}\left(\beta_{1}+\gamma_{1}+1\right) \\
& \times\left(\int_{Q(2 R)}|d u|^{n r /(n+r-1)} d x\right)^{(n+r-1) / n} \\
& +2 C(p-r) \tau\left(\int_{Q(2 R)}\left|f_{1}\right|^{r /(p-1)} d x\right) .
\end{aligned}
$$

Estimate of $I_{2}$. Consider (22), and let

$$
\begin{gathered}
X=d\left(\eta\left(u-u_{Q(2 R)}\right)\right), \\
Y=\eta d\left(u-u_{Q(2 R)}\right), \quad \varepsilon=r-p
\end{gathered}
$$

in Lemma 5; then, by Lemma 5, we have

$$
\begin{aligned}
|E| & \leq 2^{p-r} \frac{p-r+1}{r-p+1}\left|\left(u-u_{\mathrm{Q}(2 R)}\right) d \eta\right|^{r-p+1} \\
& \leq C\left|\left(u-u_{\mathrm{Q}(2 R)}\right) d \eta\right|^{r-p+1} .
\end{aligned}
$$

By (2), (38), Hölder's inequality, and Young's inequality,

$$
\begin{aligned}
\left|I_{2}\right| \leq & \int_{\mathrm{Q}(2 R)}|A(x, u, d u)||E| d x \\
\leq & C \int_{\mathrm{Q}(2 R)}\left(\beta_{1}|d u|^{p-1}+\gamma_{1}\left|u-u_{\mathrm{Q}(2 R)}\right|^{p-1}+f_{1}(x)\right) \\
& \times\left|\left(u-u_{\mathrm{Q}(2 R)}\right) d \eta\right|^{r-p+1} d x \\
\leq & \beta_{1} C\left(\int_{\mathrm{Q}(2 R)}|d u|^{r} d x\right)^{(p-1) / r} \\
& \times\left(\int_{\mathrm{Q}(2 R)}\left|\left(u-u_{\mathrm{Q}(2 R)}\right) d \eta\right|^{r} d x\right)^{(r-p+1) / r} \\
& +\gamma_{1} C\left(\int_{\mathrm{Q}(2 R)}\left|u-u_{\mathrm{Q}(2 R)}\right|^{r} d x\right)^{(p-1) / r} \\
& \times\left(\int_{\mathrm{Q}(2 R)}\left|\left(u-u_{\mathrm{Q}(2 R)}\right) d \eta\right|^{r} d x\right)^{(r-p+1) / r}
\end{aligned}
$$

$$
\begin{aligned}
& +C\left(\int_{Q(2 R)}\left|f_{1}\right|^{r /(p-1)} d x\right)^{(p-1) / r} \\
& \times\left(\int_{Q(2 R)}\left|\left(u-u_{Q(2 R)}\right) d \eta\right|^{r} d x\right)^{(r-p+1) / r} \\
& \leq \beta_{1} C \tau \int_{Q(2 R)}|d u|^{r} d x+\frac{\beta_{1} C \tau^{-(p-1) /(r-p+1)}}{R^{r}} \\
& +\int_{Q(2 R)}\left|u-u_{Q(2 R)}\right|^{r} d x \\
& +\gamma_{1} C \tau \int_{Q(2 R)}\left|u-u_{Q(2 R)}\right|^{r} d x \\
& +\frac{\gamma_{1} C \tau^{-(p-1) /(r-p+1)}}{R^{r}} \int_{Q(2 R)}\left|u-u_{Q(2 R)}\right|^{r} d x \\
& +C \tau \int_{Q(2 R)}\left|f_{1}\right|^{r /(p-1)} d x \\
& +\frac{C \tau^{-(p-1) /(r-p+1)}}{R^{r}} \int_{Q(2 R)}\left|u-u_{Q(2 R)}\right|^{r} d x .
\end{aligned}
$$

Combined with (28), the above inequality becomes

$$
\begin{aligned}
\left|I_{2}\right| \leq & \beta_{1} C \tau \int_{Q(2 R)}|d u|^{r} d x \\
& +C \tau \int_{Q(2 R)}\left|f_{1}\right|^{r /(p-1)} d x \\
& +\frac{C}{R^{r}}\left(\beta_{1}+\gamma_{1} \tau+\gamma_{1}+1\right) \\
& \times\left(\int_{Q(2 R)}|d u|^{n r /(n+r-1)} d x\right)^{(n+r-1) / n} .
\end{aligned}
$$

Estimate of $I_{3}$. By (2),

$$
\begin{aligned}
\left|I_{3}\right| \leq & \int_{Q(2 R)}|B(x, u, d u)|\left|\varphi-\varphi_{Q(2 R)}\right| d x \\
\leq & \int_{Q(2 R)}\left(\beta_{2}|d u|^{s}+\gamma_{2}\left|u-u_{Q(2 R)}\right|^{s}+f_{2}(x)\right) \\
& \times\left|\varphi-\varphi_{Q(2 R)}\right| d x \\
= & \beta_{2} \int_{Q(2 R)}|d u|^{s}\left|\varphi-\varphi_{Q(2 R)}\right| d x \\
& +\gamma_{2} \int_{Q(2 R)}\left|u-u_{Q(2 R)}\right|^{s}\left|\varphi-\varphi_{Q(2 R)}\right| d x \\
& +\int_{Q(2 R)} f_{2}(x)\left|\varphi-\varphi_{Q(2 R)}\right| d x \\
= & I_{31}+I_{32}+I_{33} .
\end{aligned}
$$


Journal of Applied Mathematics

7

Estimate of $I_{31}$. By Hölder's inequality, Lemma 4, and Young's inequality with $s r /(p-1)<r$, it yields

$$
\begin{aligned}
\left|I_{31}\right| \leq & \beta_{2}\left(\int_{Q(2 R)}|d u|^{s r /(p-1)} d x\right)^{(p-1) / r} \\
& \times\left(\int_{Q(2 R)}\left|\varphi-\varphi_{Q(2 R)}\right|^{r /(r-p+1)} d x\right)^{(r-p+1) / r} \\
\leq & \beta_{2} C(n, p, r) \operatorname{diam}(Q(2 R)) \\
& \times\left(\int_{Q(2 R)}|d u|^{s r /(p-1)} d x\right)^{(p-1) / r} \\
& \times\left(\int_{Q(2 R)}|d \varphi|^{r /(r-p+1)} d x\right)^{(r-p+1) / r} \\
\leq & \beta_{2} C(n, p, r,|\Omega|) \tau_{1} \int_{Q(2 R)}|d \varphi|^{r /(r-p+1)} d x \\
& +\beta_{2} C \tau_{2} \int_{Q(2 R)}|d u|^{r} d x+\beta_{2} C . \\
\leq & \beta_{2} C \tau_{1} \int_{Q(2 R)}|d \varphi|^{r /(r-p+1)} d x \\
& +\beta_{2} C \tau_{1}^{-(r-p+1) /(p-1)} \tau_{2} \int_{Q(2 R)}|d u|^{r} d x \\
\leq & \beta_{2} C(n, p, r,|\Omega|) \tau_{1}^{-(r-p+1) /(p-1)} \int_{Q(2 R)}|d u|^{s r /(p-1)} d x \\
& +\beta_{2} C(n, p, r,|\Omega|) \tau_{1}^{-(r-p+1) /(p-1)} \tau_{2} \int_{Q(2 R)}|d u|^{r} d x \\
& |d \varphi|^{r /(r-p+1)} d x
\end{aligned}
$$

By (21) and (28),

$$
\begin{aligned}
& \|d \varphi\|_{r /(r-p+1)}^{r /(r-p+1)} \\
& \leq C(n)\left\|d\left(\eta\left(u-u_{\mathrm{Q}(2 R)}\right)\right)\right\|_{r}^{r} \\
& \leq C(n)\left\|\eta d u+\left(u-u_{\mathrm{Q}(2 R)}\right) d \eta\right\|_{r}^{r} \\
& \leq C(n)\left(\|\eta d u\|_{r}^{r}+\left\|\left(u-u_{\mathrm{Q}(2 R)}\right) d \eta\right\|_{r}^{r}\right) \\
& \leq C(n, p, r)\left(\|d u\|_{r}^{r}+\frac{C(n)}{R^{r}}\left\|u-u_{\mathrm{Q}(2 R)}\right\|_{r}^{r}\right) \\
& \leq C\|d u\|_{r}^{r}+\frac{C}{R^{r}}\left(\int_{\mathrm{Q}(2 R)}|d u|^{n r /(n+r-1)} d x\right)^{(n+r-1) / n} .
\end{aligned}
$$

Then, combining (42) and (43),

$$
\begin{aligned}
\left|I_{31}\right| \leq & \beta_{2} C\left(\tau_{1}+\tau_{2}\right) \int_{Q(2 R)}|d u|^{r} d x \\
& +\frac{\beta_{2} C \tau_{1}}{R^{r}}\left(\int_{Q(2 R)}|d u|^{n r /(n+r-1)} d x\right)^{(n+r-1) / n}+\beta_{2} C .
\end{aligned}
$$

Estimate of $I_{32}$. Similarly, by Hölder's inequality, Lemma 4, Young's inequality, (43), and (33), it yields

$$
\begin{aligned}
& \left|I_{32}\right| \leq \gamma_{2}\left(\int_{Q(2 R)}\left|u-u_{Q(2 R)}\right|^{s r /(p-1)} d x\right)^{(p-1) / r} \\
& \times\left(\int_{\mathrm{Q}(2 R)}\left|\varphi-\varphi_{\mathrm{Q}(2 R)}\right|^{r /(r-p+1)} d x\right)^{(r-p+1) / r} \\
& \leq \gamma_{2} C(n, p, r) \operatorname{diam}(Q(2 R)) \\
& \times\left(\int_{\mathrm{Q}(2 R)}\left|u-u_{\mathrm{Q}(2 R)}\right|^{s r /(p-1)} d x\right)^{(p-1) / r} \\
& \times\left(\int_{Q(2 R)}|d \varphi|^{r /(r-p+1)} d x\right)^{(r-p+1) / r} \\
& \leq \gamma_{2} C(n, p, r,|\Omega|) \tau_{1} \int_{Q(2 R)}|d \varphi|^{r /(r-p+1)} d x \\
& +\gamma_{2} C(n, p, r,|\Omega|) \tau_{1}^{-(r-p+1) /(p-1)} \\
& \times \int_{Q(2 R)}\left|u-u_{Q(2 R)}\right|^{s r /(p-1)} d x \\
& \leq \gamma_{2} C(n, p, r,|\Omega|) \tau_{1} \int_{Q(2 R)}|d \varphi|^{r /(r-p+1)} d x \\
& +\gamma_{2} C(n, p, r,|\Omega|) \tau_{1}^{-(r-p+1) /(p-1)} \tau_{2} \\
& \times \int_{Q(2 R)}\left|u-u_{Q(2 R)}\right|^{r} d x \\
& +\gamma_{2} C(n, p, r,|\Omega|) \tau_{1}^{-(r-p+1) /(p-1)} \tau_{2}^{-s /(p-1-s)} \\
& \leq \gamma_{2} C(n, p, r,|\Omega|)\left(\tau_{1}+\tau_{1}^{-(r-p+1) /(p-1)} \tau_{2}\right) \\
& \times \int_{Q(2 R)}|d u|^{r} d x \\
& +\frac{\gamma_{2} C(n, p, r,|\Omega|) \tau_{1}}{R^{r}} \\
& \times\left(\int_{Q(2 R)}|d u|^{n r /(n+r-1)} d x\right)^{(n+r-1) / n} \\
& +\gamma_{2} C(n, p, r,|\Omega|) \tau_{1}^{-(r-p+1) /(p-1)} \tau_{2}^{-s /(p-1-s)}
\end{aligned}
$$




$$
\begin{aligned}
\leq & \gamma_{2} C\left(\tau_{1}+\tau_{2}\right) \int_{Q(2 R)}|d u|^{r} d x \\
& +\frac{\gamma_{2} C \tau_{1}}{R^{r}}\left(\int_{Q(2 R)}|d u|^{n r /(n+r-1)} d x\right)^{(n+r-1) / n}+\gamma_{2} C .
\end{aligned}
$$

Estimate of $I_{33}$. Consider

$$
\begin{aligned}
& \left|I_{33}\right|=\int_{Q(2 R)} f_{2}(x)\left|\varphi-\varphi_{Q(2 R)}\right| d x \\
& \leq\left(\int_{Q(2 R)} f_{2}^{r /(p-1)} d x\right)^{(p-1) / r} \\
& \times\left(\int_{\mathrm{Q}(2 R)}\left|\varphi-\varphi_{\mathrm{Q}(2 R)}\right|^{r /(r-p+1)} d x\right)^{(r-p+1) / r} \\
& \leq C(n, r) \operatorname{diam}(Q(2 R))\left(\int_{Q(2 R)} f_{2}^{r /(p-1)} d x\right)^{(p-1) / r} \\
& \times\left(\int_{Q(2 R)}|d \varphi|^{r /(r-p+1)} d x\right)^{(r-p+1) / r} \\
& \leq C(n, r,|\Omega|)\left(\int_{Q(2 R)} f_{2}^{r /(p-1)} d x\right)^{(p-1) / r} \\
& \times\left(\int_{\mathrm{Q}(2 R)}\left|d\left(\eta\left(u-u_{\mathrm{Q}(2 R)}\right)\right)\right|^{r} d x\right)^{(r-p+1) / r} \\
& \leq C(n, p, r,|\Omega|)\left(\int_{\mathrm{Q}(2 R)} f_{2}^{r /(p-1)} d x\right)^{(p-1) / r} \\
& \times\left(\int_{Q(2 R)}|d u|^{r} d x\right)^{(r-p+1) / r} \\
& +\frac{C(n, p, r,|\Omega|)}{R^{r-p+1}}\left(\int_{Q(2 R)} f_{2}^{r /(p-1)} d x\right)^{(p-1) / r} \\
& \times\left(\int_{Q(2 R)}|d u|^{n r /(n+r-1)} d x\right)^{(n+r-1)(r-p+1) / n r} \\
& \leq C(n, p, r,|\Omega|) \tau \int_{Q(2 R)}|d u|^{r} d x \\
& +\frac{C(n, p, r,|\Omega|) \tau}{R^{r}}\left(\int_{Q(2 R)}|d u|^{n r /(n+r-1)} d x\right)^{(n+r-1) / n} \\
& +C(n, p, r,|\Omega|) 2 \tau^{-(r-p+1) /(p-1)} \int_{\mathrm{Q}(2 R)}\left|f_{2}\right|^{r /(p-1)} d x \\
& \leq C \tau \int_{Q(2 R)}|d u|^{r} d x \\
& +\frac{C \tau}{R^{r}}\left(\int_{Q(2 R)}|d u|^{n r /(n+r-1)} d x\right)^{(n+r-1) / n} \\
& +C \int_{Q(2 R)}\left|f_{2}\right|^{r /(p-1)} d x
\end{aligned}
$$

Then, combining (41), (44), (45), and (46),

$$
\begin{aligned}
\left|I_{3}\right| \leq & C\left(\beta_{2} \tau_{1}+\beta_{2} \tau_{2}+\gamma_{2} \tau_{1}+\gamma_{2} \tau_{2}+\tau\right) \\
& \times \int_{Q(2 R)}|d u|^{r} d x \\
& +\frac{C\left(\beta_{2} \tau_{1}+\gamma_{2} \tau_{1}+\tau\right)}{R^{r}} \\
& \times\left(\int_{Q(2 R)}|d u|^{n r /(n+r-1)} d x\right)^{(n+r-1) / n} \\
& +C \int_{Q(2 R)}\left|f_{2}\right|^{r /(p-1)} d x+C\left(\beta_{2}+\gamma_{2}\right) .
\end{aligned}
$$

Combining with (27), (36), (40), and (47), we get

$$
\begin{aligned}
& \alpha \int_{Q(R)}|d u|^{r} d x \\
& \leq C\left((p-r)\left(\beta_{1}+\beta_{1} \tau+\gamma_{1}+\gamma_{1} \tau+1\right)\right. \\
&\left.+\left(\beta_{1} \tau+\beta_{2} \tau_{1}+\beta_{2} \tau_{2}+\gamma_{2} \tau_{1}+\gamma_{2} \tau_{2}+\tau\right)\right) \\
& \times \int_{Q(2 R)}|d u|^{r} d x \\
&+\frac{C}{R^{r}}\left((p-r)\left(\beta_{1}+\gamma_{1}+1\right)+\left(\beta_{1}+\gamma_{1} \tau+\gamma_{1}+1\right)\right. \\
&+\left(\int_{Q(2 R)}|d u|^{n r /(n+r-1)} d x\right)^{(n+r-1) / n} \\
&+(2 C(p-r) \tau+C \tau) \\
& \times \int_{Q(2 R)}\left|f_{1}\right|^{r /(p-1)} d x \\
&+C \int_{Q(2 R)}\left|f_{2}\right|^{r /(p-1)} d x+C\left(\beta_{2}+\gamma_{2}\right)
\end{aligned}
$$

Step 3. A higher integrability is obtained by a weak reverse Hölder inequality. Now, we are in a position to take $r$ sufficiently close to $p$, such that $(p-r)\left(\beta_{1}+\beta_{1} \tau+\gamma_{1}+\gamma_{1} \tau+1\right)<$ $\min \{\alpha / 8 C, 1 / 2\}$, and take $\tau, \tau_{1}$, and $\tau_{2}$ small enough such that $\beta_{1} \tau+\beta_{2} \tau_{1}+\beta_{2} \tau_{2}+\gamma_{2} \tau_{1}+\gamma_{2} \tau_{2}+\tau<\alpha / 8 C$. Then, the summation of the coefficients of the first term in the right-hand side of (48) is smaller than $\alpha$. This implies

$$
\begin{aligned}
f_{\mathrm{Q}(R)}|d u|^{r} d x \leq & \theta f_{\mathrm{Q}(2 R)}|d u|^{r} d x \\
& +C\left(\int_{\mathrm{Q}(2 R)}|d u|^{n r /(n+r-1)} d x\right)^{(n+r-1) / n} \\
& +C f_{\mathrm{Q}(2 R)}\left(\left|f_{1}\right|^{r /(p-1)}+\left|f_{2}\right|^{r /(p-1)}+1\right) d x .
\end{aligned}
$$


Setting $g=|d u|^{n r /(n+r-1)}, q=(n+r-1) / n$, and $f=C\left(\left|f_{1}\right|^{r /(p-1)}+\left|f_{2}\right|^{r /(p-1)}+1\right)^{n /(n+r-1)}$, we obtain from Lemma 7 that

$$
\begin{aligned}
& \left(f_{\mathrm{Q}(R)}|d u|^{r^{\prime}} d x\right)^{1 / r^{\prime}} \\
& \leq C\left(f_{\mathrm{Q}(2 R)}|d u|^{r} d x\right)^{1 / r} \\
& \quad+C\left(f_{\mathrm{Q}(2 R)}\left(\left|f_{1}\right|^{r /(p-1)}+\left|f_{2}\right|^{r /(p-1)}+1\right)^{r^{\prime} / r} d x\right)^{1 / r^{\prime}}
\end{aligned}
$$

for some $r^{\prime}=r^{\prime}(p, s, n, \alpha / \beta)>r$. The above inequality implies that $d u$ satisfies a weak reverse Hölder inequality. The integrability exponent of $d u$ has improved from $r=p-\varepsilon_{0}$ to $r^{\prime}=p-\varepsilon_{0}+\varepsilon_{1}$.

We are now in a position to repeatedly use Lemma 7 to improve the degree of integrability of $d u$ that allows us to increase the exponent $r^{\prime}$ even further. This idea can trace from a series of works of Iwaniec and his coworkers $[3,18$, 20]. Reasoning as before $u \in W_{\text {loc }}^{1, r^{\prime \prime}}\left(\Omega, \bigwedge^{l-1}\right)$ with some $r^{\prime \prime}>r^{\prime}$ and the reverse Hölder inequality (50) remains valid with the exponent $r^{\prime \prime}$ in place of $r^{\prime}$. We improve the degree of integrability of $\nabla u$ again and again. It is clear that one can reach any number $q \in\left(r_{1}, r_{2}\right)$ to conclude with $u \in$ $W_{\text {loc }}^{1, q}\left(\Omega, \bigwedge^{l-1}\right)$.

\section{Conflict of Interests}

The authors declare that there is no conflict of interests regarding the publication of this paper.

\section{Acknowledgments}

The authors are supported by NSFC (11371050) and NSF of Hebei Province (A2013209278). The authors would like to thank the referee of this paper for helpful suggestions.

\section{References}

[1] R. P. Agarwal, S. Ding, and C. Nolder, Inequalities for Differential Forms, Springer, New York, NY, USA, 2009.

[2] C. A. Nolder, "Hardy-Littlewood theorems for A-harmonic tensors," Illinois Journal of Mathematics, vol. 43, no. 4, pp. 613632, 1999.

[3] T. Iwaniec and C. Sbordone, "Weak minima of variational integrals," Journal für die Reine und Angewandte Mathematik, vol. 454, pp. 143-161, 1994.

[4] S. Ding and J. Zhu, "Poincaré-type inequalities for the homotopy operator with $L^{\phi}(\Omega)$-norms," Nonlinear Analysis: Theory, Methods \& Applications, vol. 74, no. 11, pp. 3728-3735, 2011.

[5] S. Ding and C. A. Nolder, "Weighted poincaré inequalities for solutions to A-harmonic equations," Illinois Journal of Mathematics, vol. 46, no. 1, pp. 199-205, 2002.
[6] Y. Wang and G. Li, "Weighted decomposition estimates for differential forms," Journal of Inequalities and Applications, vol. 2010, Article ID 649340, 17 pages, 2010.

[7] Y. Xing, "Poincaré inequalities with Luxemburg norms in $L^{\prime}(m)$-averaging domains," Journal of Inequalities and Applications, vol. 2010, Article ID 241759, 11 pages, 2010.

[8] H. Gao, "Weighted integral inequalities for conjugate $A$ harmonic tensors," Journal of Mathematical Analysis and Applications, vol. 281, no. 1, pp. 253-263, 2003.

[9] J. Heinonen, T. Kilpelainen, and O. Martio, Nonlinear Potential Theory Of Degenerate Elliptic Equations, Oxford University Press, New York, NY. USA, 1993.

[10] G. Li and C. Yang, "The existence of a nontrivial solution to a nonlinear elliptic boundary value problem of p-Laplacian type without the Ambrosetti-Rabinowitz condition," Nonlinear Analysis, Theory, Methods and Applications, vol. 72, no. 12, pp. 4602-4613, 2010.

[11] S. Zheng, X. Zheng, and Z. Feng, "Optimal regularity for Aharmonic type equations under the natural growth," Discrete and Continuous Dynamical Systems B, vol. 16, no. 2, pp. 669685, 2011.

[12] H. Gao, J. Qiao, and Y. Chu, "Local regularity and local boundedness results for very weak solutions of obstacle problems," Journal of Inequalities and Applications, vol. 2010, Article ID 878769, 12 pages, 2010.

[13] G. Bao, T. Wang, and G. Li, "On very weak solutions to a class of double obstacle problems," Journal of Mathematical Analysis and Applications, vol. 402, no. 2, pp. 702-709, 2013.

[14] B. Stroffolini, "On weakly A-harmonic tensors," Studia Mathematica, vol. 114, no. 3, pp. 289-301, 1995.

[15] H. Gao and Y. Wang, "Weak $W T_{2}$-class of differential forms and weakly A-harmonic tensors," Applied Mathematics-A Journal of Chinese Universities, vol. 25, no. 3, pp. 359-366, 2010.

[16] T. Iwaniec and A. Lutoborski, "Integral estimates for null Lagrangians," Archive for Rational Mechanics and Analysis, vol. 125, no. 1, pp. 25-79, 1993.

[17] T. Iwaniec and G. Martin, "Quasiregular mappings in even dimensions," Acta Mathematica, vol. 170, no. 1, pp. 29-81, 1993.

[18] T. Iwaniec, L. Migliaccio, L. Nania, and C. Sbordone, "Integrability and removability results for quasiregular mappings in high dimensions," Mathematica Scandinavica, vol. 75, no. 2, pp. 263-279, 1994.

[19] M. Giaquinta, Multiple Integrals in the Calculus of Variations and Nonlinear Elliptic Systems, vol. 105 of Annals of Mathematics Studies, Princeton University Press, Princeton, NJ, USA, 1983.

[20] T. Iwaniec, "p-Harmonic tensors and quasiregular mappings," Annals of Mathematics. Second Series, vol. 136, no. 3, pp. 589624, 1992. 


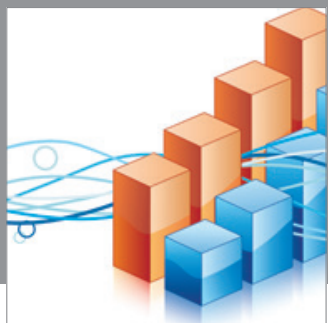

Advances in

Operations Research

mansans

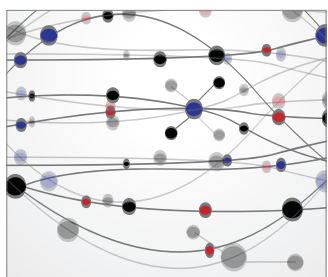

The Scientific World Journal
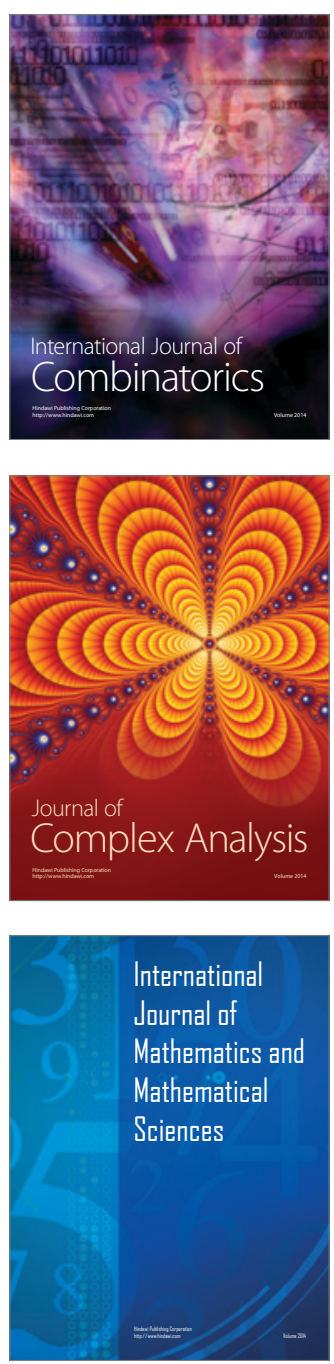
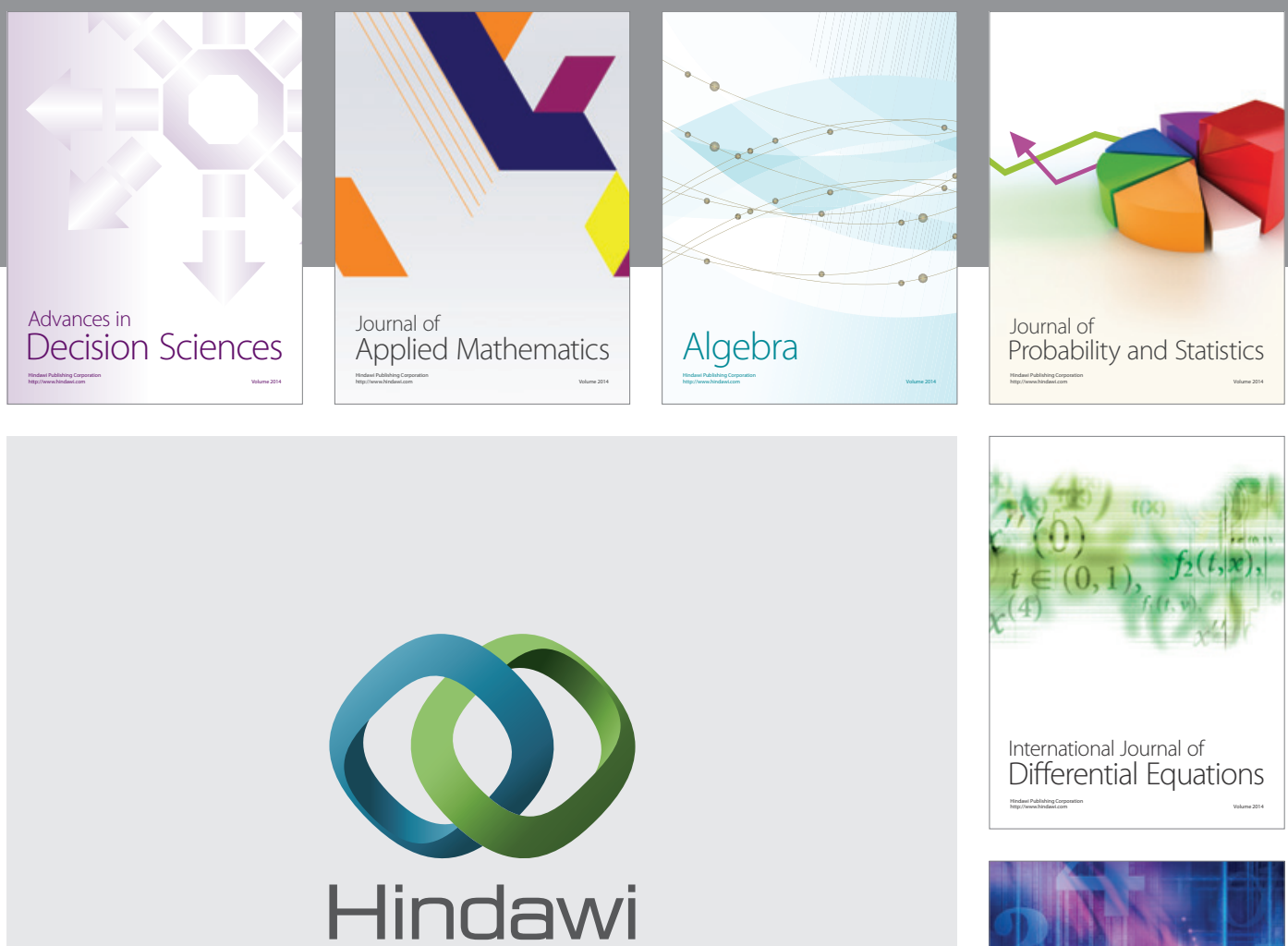

Submit your manuscripts at http://www.hindawi.com
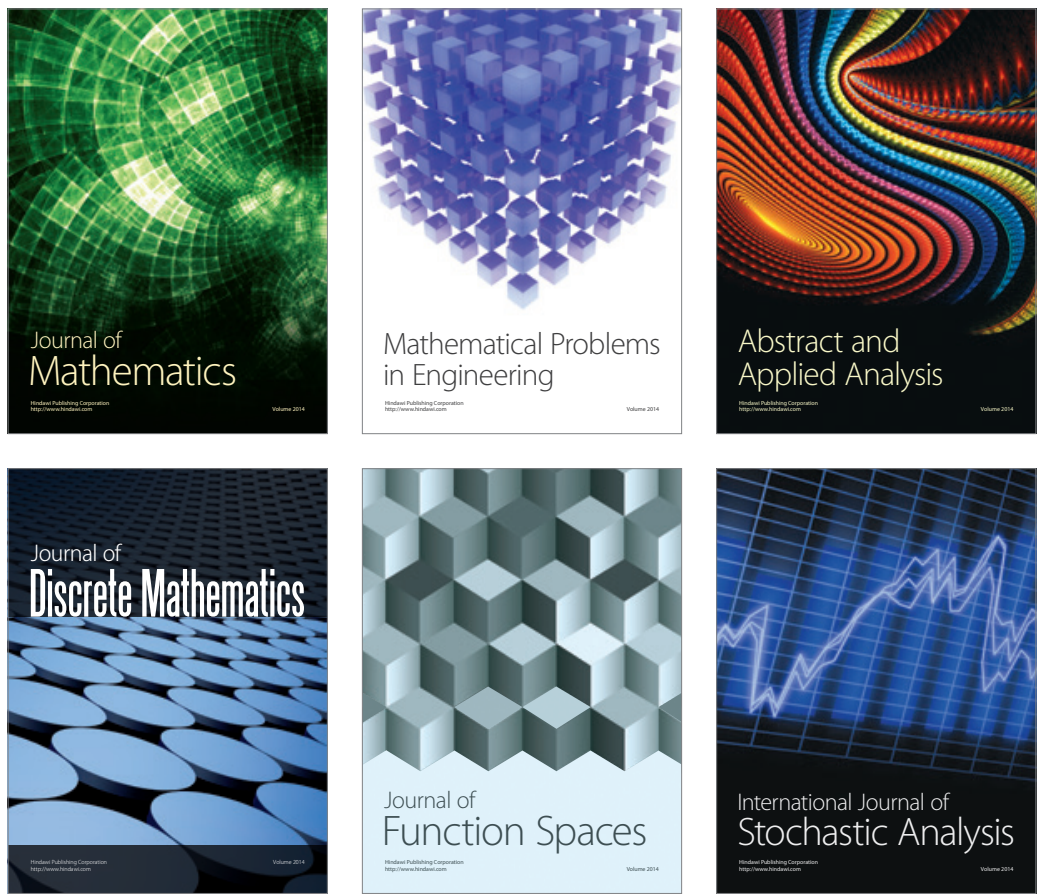

Journal of

Function Spaces

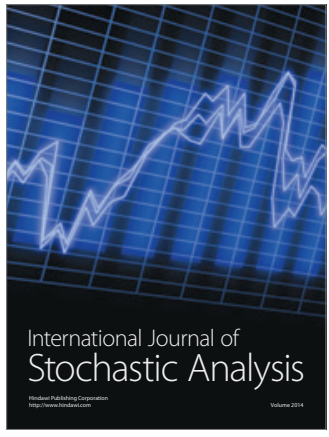

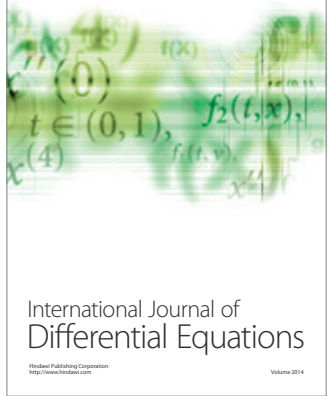
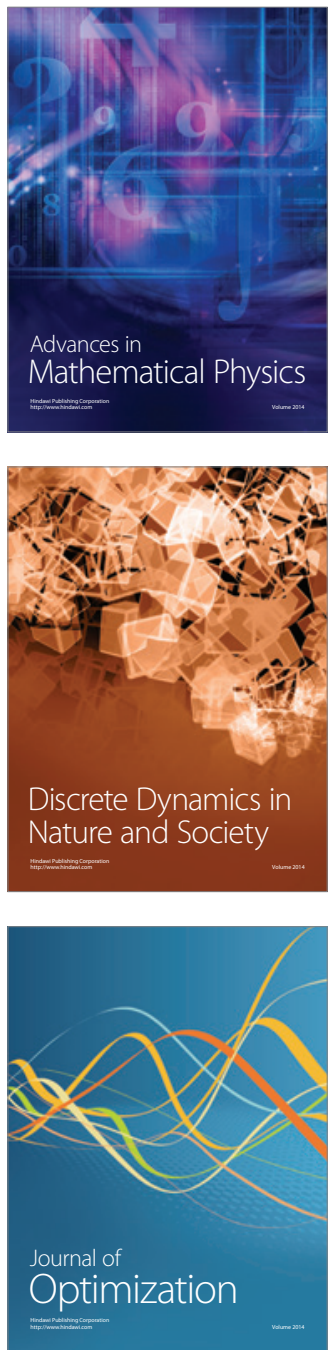